\title{
THREE PRINCIPLES LEADING TO THE BELL INEQUALITIES
}

\begin{abstract}
In the paper we compare three principles accounting for correlations, namely Reichenbach's Common Cause Principle, Bell's Local Causality Principle, and Einstein's Reality Criterion and relate them to the Bell inequalities. We show that there are two routes connecting the principles to the Bell inequalities. In case of Reichenbach's Common Cause Principle and Bell's Local Causality Principle one assumes a non-conspiratorial joint common cause for a set of correlations. In case of Einstein's Reality Criterion one assumes strongly nonconspiratorial separate common causes for a set of perfect correlations. Strongly non-conspiratorial separate common causes for perfect correlations, however, form a non-conspiratorial joint common cause. Hence the two routes leading the Bell inequalities meet.
\end{abstract}

Keywords: Einstein's reality criterion, Common Cause Principle, local causality, Bell inequalities

\section{Introduction}

Many were pondering on the historical reasons of why it took thirty years to get from EPR argument to the Bell inequalities (see, for example: Bell 1964/2004; Howard 1985; Redhead 1987; Hájek and Bub 1992; Fine 1996; Norton 2004; Szabó 2008; Goldstein et al. 2011; Maudlin 2014; Lewis 2015). This paper has nothing to say about these historical and conceptual reasons. It rather intends to show that the route leading from Einstein's Reality Criterion to the Bell inequalities is no longer than the route starting off from two other principles standardly used to causally account for correlations, namely Reichenbach's Common Cause Principle and Bell's Local Causality Principle.

In the paper we will handle the three principles side by side and show how they relate to one another and to the Bell inequalities. In Section 2 we show how the principles are used to causally account for correlations; in Section 3 we use them to explain conditional correlations; and in Section 4 we trace the routes leading from the principles to the Bell inequalities. In the paper we deliberately keep the philosophical analysis short so that the formal parallelism will not be lost sight of.

\footnotetext{
* Research Center for the Humanities, Budapest; email: szabo.gabor@btk.mta.hu
} 


\section{Explaining correlations}

Let $A$ and $B$ be two correlated but causally separated events represented in a classical probability space $(\Sigma, p)$ :

$$
p(A \wedge B) \neq p(A) p(B)
$$

One can invoke three principles to causally account for this correlation. If one is concerned only with the probabilistic aspects, one can apply

Reichenbach's Common Cause Principle: If there is a correlation between two events and there is no direct causal (or logical) connection between the correlated events, then there always exists a common cause of the correlation.

Formally, a common cause of the correlation (1) is a partition $\left\{C_{k}\right\}(k \in K)$ in $(\Sigma, p)$ - or in an extension of $(\Sigma, p)$; see Hofer-Szabó, Rédei and Szabó 2013such that for any $k \in K$ :

$$
p\left(A \wedge B \mid C_{k}\right)=p\left(A \mid C_{k}\right) p\left(B \mid C_{k}\right)
$$

If one furthermore assumes that the events $A$ and $B$ also have spatiotemporal localization, for example they are located in spatially separated regions, $V_{A}$ and $V_{B}$, respectively, then to causally account for them, one can invoke a further principle:

Bell's Local Causality Principle: "A theory will be said to be locally causal if the probabilities attached to values of local beables in a space-time region $V_{A}$ are unaltered by specification of values of local beables in a spatially separated region $V_{B}$, when what happens in the backward light cone of $V_{A}$ is already sufficiently specified, for example by a full specification of local beables in a space-time region $V_{C}^{\prime \prime}$ (Bell 1990/2004, 239-240).

The figure Bell is attaching to this formulation is reproduced in Fig. 1 with the original caption. In a locally causal theory for any correlation between events $A$ and $B$ localized in spatially separated regions $V_{A}$ and $V_{B}$, respectively, the atomic partition $\left\{C_{k}\right\}(k \in K)$ in the probability space $(\Sigma, p)$ associated to any region $V_{C}$ causally shielding-off $V_{A}$ from the common past of $V_{A}$ and $V_{B}$ as depicted Fig. 1 should satisfy (2).

Finally, suppose we interpret the correlation (1) epistemologically as a prediction. That is we interpret $A$ as a predicting event and $B$ as a predicted event and the prediction as a correlation between the two. After all, a prediction is ontologically nothing but an (ideally strong) correlation between two event types. Weather forecast is simply a correlation between the today announcement and the tomorrow weather. Moreover, in a prediction the predicted event cannot causally influence the predicting events. One can predict the tomorrow weather but not the yesterday weather.

Suppose furthermore that the following two requirements also hold: (i) The predicting event is also causally irrelevant for the predicted event. This can happen for example when 


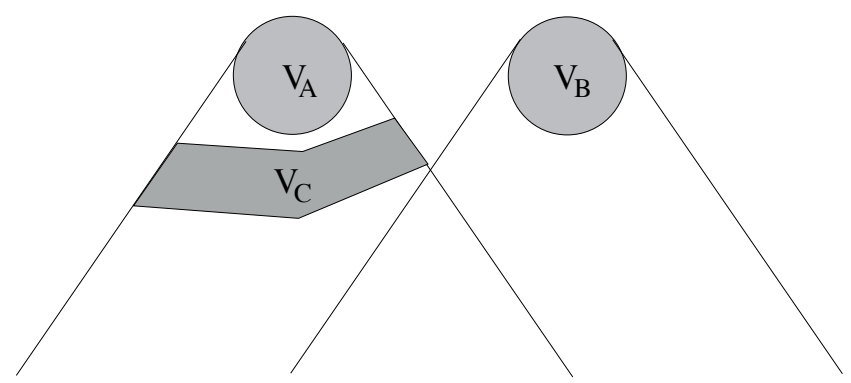

Figure 1: Full specification of what happens in $V_{C}$ makes events in $V_{B}$ irrelevant for predictions about $V_{A}$ in a locally causal theory.

the two events are spatially separated. (ii) The correlation between $A$ and $B$ is perfect:

$$
p(A \wedge B)=p(A)=p(B)
$$

If all these hold, then we have a third principle to account for the correlation (3):

Einstein's Reality Criterion: "If, without in any way disturbing a system, we can predict with certainty (i.e. with probability equal to unity) the value of a physical quantity, then there exists an element of physical reality corresponding to this physical quantity" (Einstein, Podolsky, and Rosen 1935, 777-778).

Observe, that the term "without in any way disturbing a system" is just condition (i) above, and the term "predict with certainty" is just condition (ii). What Einstein's Reality Criterion requires is that in case of a perfect prediction, that is perfect correlation between causally separated events, an element of reality should account for the correlation.

What is an element of reality?

The distinctive feature of an element of reality (see Gömöri and Hofer-Szabó 2017 for the details) is that it determines the predicted event with certainty. Formally, an element of reality is a partition $\left\{C^{+}, C^{-}\right\}$in $(\Sigma, p)$ such that the following holds:

$$
\begin{aligned}
& p\left(A \wedge B \mid C^{+}\right)=1 \\
& p\left(A \wedge B \mid C^{-}\right)=0
\end{aligned}
$$

Now, let us go back to the Reichenbach's Common Cause Principle. It is well known that for perfect correlations a common cause that is a partition $\left\{C_{k}\right\}(k \in K)$ satisfying (2) is deterministic: for any $k \in K$

$$
p\left(A \wedge B \mid C_{k}\right) \in\{0,1\}
$$

Hence, the indices $k \in K$ can be grouped into two groups $K^{+}$and $K^{-}$with $K^{+} \vee K^{-}$ $=K$ such that

$$
\begin{aligned}
& C^{+}=\vee_{k \in K^{+}} C_{k} \\
& C^{-}=v_{k \in K^{-}} C_{k}
\end{aligned}
$$


and $\left\{C^{+}, C^{-}\right\}$satisfies (4)-(5). Common causes for perfect correlations understood as predictions are just elements of reality.

To sum up, a correlation between two events depending on whether we understand it purely probabilistically or spatiotemporally or in the context of predictions can be explained by three different principles: by Reichenbach's Common Cause Principle, by Bell's Local Causality Principle or by Einstein's Reality Criterion.

\section{Explaining conditional correlations}

Now, let us apply the above reasoning to measurements. Let $a_{i}$ and $b_{j}(i \in I, j \in$ $J$ ) be measurement choices and let $\left\{A_{i}, A_{i}^{\prime}\right\}$ and $\left\{B_{i}, B_{j}^{\prime}\right\}$ be binary measurement outcomes on two spatially separated systems. We will represent the measurement choices as two partitions $\left\{a_{i}\right\}(i \in I)$ and $\left\{b_{j}\right\}(j \in J)$ in a classical probability space $(\Sigma, p)$, and the measurement outcomes by further partitioning the appropriate measurement choices $a_{i}$ and $b_{j}$, respectively:

$$
\begin{array}{ll}
A_{i} \wedge A_{i}^{\prime}=0 & A_{i} \vee A_{i}^{\prime}=a_{i} \\
B_{j} \wedge B_{j}^{\prime}=0 & B_{j} \vee B_{j}^{\prime}=b_{j}
\end{array}
$$

Suppose that for a given $i \in I$ and $j \in J$ the measurement outcomes $A_{i}$ and $B_{j}$ are conditionally correlated in the following sense:

$$
p\left(A_{i} \wedge B_{j} \mid a_{i} \wedge b_{j}\right) \neq p\left(A_{i} \mid a_{i}\right) p\left(B_{j} \mid b_{j}\right)
$$

What is the causal explanation of this conditional correlation?

Before we turn to the above principles, we make the following stipulation: Whatever explains the above correlations, it has to be causally and hence probabilistically independent of the measurement choices. In other words, in applying the above principles we will always require:

No-conspiracy: If a partition $\left\{C_{k}\right\}(k \in K)$ represents a set of events explaining the correlation (11), then for any $k \in K$ the following relation is required:

$$
p\left(a_{i} \wedge b_{j} \wedge C_{k}\right)=p\left(a_{i} \wedge b_{j}\right) p\left(C_{k}\right)
$$

Next, we formulate the three principles causally accounting for the conditional correlation between the measurement outcomes given certain measurement choices:

Reichenbach's Common Cause Principle: The common cause of the conditional correlation (11) is a partition $\left\{C_{k}\right\}$ in $(\Sigma, p)$ such that for any $k \in K$ :

$$
\begin{aligned}
p\left(A_{i} \wedge B_{j} \mid a_{i} \wedge b_{j} \wedge C_{k}\right) & =p\left(A_{i} \mid a_{i} \wedge C_{k}\right) p\left(B_{j} \mid b_{j} \wedge C_{k}\right) \\
p\left(a_{i} \wedge b_{j} \wedge C_{k}\right) & =p\left(a_{i} \wedge b_{j}\right) p\left(C_{k}\right)
\end{aligned}
$$


Bell's Local Causality Principle: Suppose there is a conditional correlation (11) between measurement outcomes $A_{i}$ and $B_{j}$ given measurement choices $a_{i}$ and $b_{j}$. Suppose further that $A_{i}$ and $a_{i}$ are localized in regions $V_{A}$ and $B_{j}$ and $b_{j}$ are localized in regions $V_{B}$ spatially separated from $V_{A}$. Then, if the theory accounting for this correlation is locally causal, then the atomic partition $\left\{C_{k}\right\}$ ( $k$ $\in K)$ in $(\Sigma, p)$ associated to the region $V_{C}$ (see Fig. 1) should satisfy (13)-(14).

Einstein's Reality Criterion: Suppose that the conditional correlation (11) represents now a prediction. That is let $A_{i}$ denote the outcome of a predicting event $a_{i}$ and let $B_{j}$ denote the outcome of the predicted event $b_{j}$. Suppose furthermore that $A_{i}, a_{i}$ and $B_{j}, b_{j}$ are causally separated. Also suppose that we can predict the outcome $B_{j}$ of the measurement $b_{j}$ by obtaining outcome $A_{i}$ for the prediction $a_{i}$ for sure. In other words, suppose that the conditional correlation is perfect:

$$
p\left(A_{i} \wedge B_{j} \mid a_{i} \wedge b_{j}\right)=p\left(A_{i} \mid a_{i}\right)=p\left(B_{j} \mid b_{j}\right)
$$

Then Einstein's Reality Criterion claims that there are elements of reality that is a partition $\left\{C^{+}, C^{-}\right\}$in $(\Sigma, p)$ explaining correlation (15) in the following sense:

$$
\begin{aligned}
p\left(A_{i} \wedge B_{j} \mid a_{i} \wedge b_{j} \wedge C^{+}\right) & =1 \\
p\left(A_{i} \wedge B_{j} \mid a_{i} \wedge b_{j} \wedge C^{-}\right) & =0 \\
p\left(a_{i} \wedge b_{j} \wedge C^{+}\right) & =p\left(a_{i} \wedge b_{j}\right) p\left(C^{+}\right) \\
p\left(a_{i} \wedge b_{j} \wedge C^{-}\right) & =p\left(a_{i} \wedge b_{j}\right) p\left(C^{-}\right)
\end{aligned}
$$

Just as above, in case of a perfect correlation a common cause $\left\{C_{k}\right\}(k \in K)$ satisfying (13)-(14) is deterministic, hence a suitable grouping of the $C_{k}$-s via (7)-(8) will yield the elements of reality $C^{+}$and $C^{-}$. In short, Einstein's Reality Criterion is a special case of Reichenbach's Common Cause Principle when the correlation is perfect (for the details see Gömöri and Hofer-Szabó 2017).

To sum up, the core of all three principles is to account for correlations in terms of a non-conspiratorial common cause. In case of Reichenbach's Common Cause Principle only the probabilistic aspects (13)-(14) of the common cause are taken into consideration. In case of Bell's Local Causality Principle both the correlated events and also the common cause have a spatiotemporal localization. In case of Einstein's Reality Criterion the whole correlation scenario is interpreted in the framework of a prediction and the correlation is taken to be perfect.

Before we move on to the relation of the principles to the Bell inequalities, let us see how the conditional and unconditional correlations and their explanations relate to one another.

First, observe that if the measurement choices are causally and therefore probabilistically independent, that is if for any $i \in I$ and $j \in J$ :

$$
p\left(a_{i} \wedge b_{j}\right)=p\left(a_{i}\right) p\left(b_{j}\right)
$$


and the algebraic inclusions (9)-(10) hold, then the outcomes $A_{i}$ and $B_{j}$ are correlated in the conditional sence

$$
p\left(A_{i} \wedge B_{j} \mid a_{i} \wedge b_{j}\right) \neq p\left(A_{i} \mid a_{i}\right) p\left(B_{j} \mid b_{j}\right)
$$

if and only if they are correlated in the unconditional sense

$$
p\left(A_{i} \wedge B_{j}\right) \neq p\left(A_{i}\right) p\left(B_{j}\right)
$$

Second, given (9)-(10) and (20), $\left\{C_{k}\right\}$ is a non-conspiratorial common cause of the conditional correlation (21):

$$
\begin{aligned}
p\left(A_{i} \wedge B_{j} \mid a_{i} \wedge b_{j} \wedge C_{k}\right) & =p\left(A_{i} \mid a_{i} \wedge C_{k}\right) p\left(B_{j} \mid b_{j} \wedge C_{k}\right) \\
p\left(a_{i} \wedge b_{j} \wedge C_{k}\right) & =p\left(a_{i} \wedge b_{j}\right) p\left(C_{k}\right)
\end{aligned}
$$

if and only if $\left\{C_{k}\right\}$ is a non-conspiratorial common cause of the unconditional correlation (22):

$$
\begin{aligned}
p\left(A_{i} \wedge B_{j} \mid C_{k}\right) & =p\left(A_{i} \mid C_{k}\right) p\left(B_{j} \mid C_{k}\right) \\
p\left(a_{i} \wedge b_{j} \wedge C_{k}\right) & =p\left(a_{i} \wedge b_{j}\right) p\left(C_{k}\right)
\end{aligned}
$$

(For the proof see Hofer-Szabó, Rédei and Szabó 2013, Lemma 9.8.). Therefore, on the assumptions (9)-(10) and (20), the common causal explanations (23)-(24) and (25)-(26) are interchangeable.

\section{From the principles to the Bell inequalities}

How the above three principles serving for a causal explanation of correlations relate to the Bell inequalities? The crucial point is to see how the different principles relate to the common causal explanation of more correlations. Principally, there are two possible ways: either the different correlations are explained by a joint common cause or each correlation is explained by a separate common cause. The standard derivation of the Bell inequalities from Reichenbach's Common Cause Principle and Bell's Local Causality Principle assumes a joint common cause; whereas the derivation of the Bell inequalities from Einstein's Reality Criterion assumes only separate common causes. Since the assumption of separate common causes is weaker than that of a joint common cause, the derivation of the Bell inequalities from Einstein's Reality Criterion needs a stronger version of no-conspiracy.

Let us see the derivations in turn:

Reichenbach's Common Cause Principle. Suppose that $I=J=\{1,2\}$ and the events $A_{i}$ and $B_{j}$ are all conditionally correlated that is for any $i, j \in I$ :

$$
p\left(A_{i} \wedge B_{j} \mid a_{i} \wedge b_{j}\right) \neq p\left(A_{i} \mid a_{i}\right) p\left(B_{j} \mid b_{j}\right)
$$


The four correlations are said to have a non-conspiratorial joint common cause if there is a single partition $\left\{C_{k}\right\}(k \in K)$ in $(\Sigma, p)$ (or in an extension of $\left.(\Sigma, p)\right)$ such that for all $i, j \in I$ and $k \in K$ the following hold:

$$
\begin{aligned}
p\left(A_{i} \wedge B_{j} \mid a_{i} \wedge b_{j} \wedge C_{k}\right) & =p\left(A_{i} \mid a_{i} \wedge C_{k}\right) p\left(B_{j} \mid b_{j} \wedge C_{k}\right) \\
p\left(a_{i} \wedge b_{j} \wedge C_{k}\right) & =p\left(a_{i} \wedge b_{j}\right) p\left(C_{k}\right)
\end{aligned}
$$

We claim that the events $A_{i}, B_{j}, a_{i}$ and $b_{j}$ with a non-conspiratorial joint common causal explanation satisfy the Clauser-Horne inequalities that is for any $i, i^{\prime}, j, j^{\prime} \in I$ and $i \neq i^{\prime}, j \neq j^{\prime}$ :

$$
\begin{aligned}
& -1 \leqslant p\left(A_{i} \wedge B_{j} \mid a_{i} \wedge b_{j}\right)+p\left(A_{i} \wedge B_{j^{\prime}} \mid a_{i} \wedge b_{j^{\prime}}\right) \\
& +p\left(A_{i^{\prime}} \wedge B_{j} \mid a_{i^{\prime}} \wedge b_{j}\right)-p\left(A_{i^{\prime}} \wedge B_{j^{\prime}} \mid a_{i^{\prime}} \wedge b_{j^{\prime}}\right) \\
& -p\left(A_{i} \mid a_{i}\right)-p\left(B_{j} \mid b_{j}\right) \leqslant 0
\end{aligned}
$$

For the proof see the Appendix.

Bell's Local Causality Principle. Again, let $I=J=\{1,2\}$. Suppose that the events $A_{i}$ and $B_{i}$ localized in spatially separated regions $V A$ and $V_{B}$ respectively, are all conditionally correlated in the sence of (27). In a locally causal theory the atomic partition of the local algebra associated to $V_{C}$ (see again Fig. 1) is a nonconspiratorial joint common cause in the sense of (28)-(29). Hence the ClauserHorne inequalities (30) follow, just as in the case of Reichenbach's Common Cause Principle.

Einstein's Reality Criterion. Suppose now that $I=J=\{1,2,3,4\}$ and there is a perfect conditional correlation between (the predicting events) $A_{i}$ and (the predicted events) $B_{j}$ for any $i=j \in \mathrm{I}$ :

$$
p\left(A_{i} \wedge B_{i} \mid a_{i} \wedge b_{i}\right)=p\left(A_{i} \mid a_{i}\right)=p\left(B_{i} \mid b_{i}\right)
$$

First, observe that the four correlations in (31) are not the same as the correlations (27) above. In (27) $I=J=\{1,2\}$ and the four correlations were not necessarily perfect; in (31) $I=J=\{1,2,3,4\}$ and the four correlations are the $i=j$ perfect correlations.

Now, Einstein's Reality Criterion does not assume that all four correlations in (31) have a joint common cause. All it assumes is that there are separate elements of reality to each correlation, that is for any $i \in I$ there is a partiton $\left\{C_{i}^{+}, C_{i}^{-}\right\}$satisfying

$$
\begin{aligned}
& p\left(A_{i} \wedge B_{i} \mid a_{i} \wedge b_{i} \wedge C_{i}^{+}\right)=1 \\
& p\left(A_{i} \wedge B_{i} \mid a_{i} \wedge b_{i} \wedge C_{i}^{-}\right)=0
\end{aligned}
$$

However, instead of simply requiring no-conspiracy:

$$
\begin{aligned}
& p\left(a_{i} \wedge b_{j} \wedge C_{k}^{+}\right)=p\left(a_{i} \wedge b_{j}\right) p\left(C_{k}^{+}\right) \\
& p\left(a_{i} \wedge b_{j} \wedge C_{k}^{-}\right)=p\left(a_{i} \wedge b_{j}\right) p\left(C_{k}^{-}\right)
\end{aligned}
$$


$(i, j, k \in I)$ one requires strong no-conspiracy, namely that any element $C$ in the Boolean algebra generated by the four pairs of elements of reality $\left\{C_{k}^{ \pm}\right\}$should be independent of any combination of the measurement choices:

$$
p\left(a_{i} \wedge b_{j} \wedge C\right)=p\left(a_{i} \wedge b_{j}\right) p(C)
$$

In short, in case of more correlations Einstein's Reality Criterion requires less than the other two principles since it requires only separate elements of reality for the different correlations, but also requires more since it requires all Boolean combinations of the elements of reality to be independent of the measurement choices.

The derivation of the Clauser-Horne inequalities (30) from a strongly nonconspiratorial separate common casual explanation is straightforward. From (31), (32)-(33) and (36) it follows that for any $i, j \in I$ :

$$
\begin{aligned}
& p\left(A_{i} \mid a_{i}\right)=p\left(B_{i} \mid b_{i}\right)=p\left(C_{i}^{+}\right) \\
& p\left(A_{i} \wedge B_{j} \mid a_{i} \wedge b_{j}\right)=p\left(C_{i}^{+} \wedge C_{j}^{+}\right)
\end{aligned}
$$

Now, it is an elementary fact of classical probability theory that for any four events $C_{i}^{+}, C_{i^{\prime}}^{+}, C_{j}^{+}$and $C_{j^{\prime}}^{+}$in $(\Sigma, p)$ we have:

$$
\begin{aligned}
& -1 \leqslant p\left(C_{i}^{+} \wedge C_{j}^{+}\right)+p\left(C_{i}^{+} \wedge C_{j^{\prime}}^{+}\right)+p\left(C_{i^{\prime}}^{+} \wedge C_{j}^{+}\right) \\
& -p\left(C_{i^{\prime}}^{+} \wedge C_{j^{\prime}}^{+}\right)-p\left(C_{i}^{+}\right)-p\left(C_{j}^{+}\right) \leqslant 0
\end{aligned}
$$

Substituting (37)-(38) into (39) one arrives at (30).

What one proves here is that the atomic partition composed of the intersections of strongly non-conspiratorial separate common causes for perfect correlations form a non-conspiratorial joint common cause for all correlations. Note that in the general case that is for non-perfect correlations the relation between separate and joint common causes is not so straightforward and the relation of strongly nonconspiratorial separate common causes to the Bell inequalities is not known (see Hofer-Szabó, Rédei and Szabó 2013, Conjecture 9.11.).

To sum up, one can arrive at the Bell inequalities from the three principles on two different routes. In the standard derivation based on Reichenbach's Common Cause Principle or Bell's Local Causality Principle one takes four correlations and assumes that they have a non-conspiratorial joint common cause. In case of Einstein's Reality Criterion one takes four perfect correlations and assumes that each has a separate common cause which together are strongly non-conspiratorial. Both routes lead directly to the Clauser-Horne inequalities.

\section{Conclusions}

In this paper we compared three principles accounting for correlations and related them to the Bell inequalities. Reichenbach's Common Cause Principle, in the original sense at least, refers only to one correlation: it demands a common cause for a given correlation if the direct causal link between the 
correlata can be excluded. In the derivation of the Bell inequalities, however, the principle had to be used in a stronger sense, namely demanding one and the same cause for a set of correlations. Bell's Local Causality Principle has already been formulated originally in this strong sense: all correlations localized in spatially separated regions were to be screened-off by the "full specification" of an appropriately localized third spacetime region. In this sense Bell's Local Causality Principle is a stronger principle than Reichenbach's Common Cause Principle. Finally, Einstein's Reality Criterion again assumes elements of reality to each correlation separately, similar to Reichenbach's Common Cause Principle. Moreover, it does so only in case of correlations. In this sense Einstein's Reality Criterion seems to be even weaker than Reichenbach's Common Cause Principle.

Note, however, that not even the strongest of the three principles, namely Bell's Local Causality Principle implies the Bell inequalities on its own. Even this principle needs to assume that the common causes or elements of reality causally responsible for the correlations are causally and hence probabilistically independent from the measurement choices. To be sure, no-conspiracy seems to be a natural requirement for an element of reality to deserve its name. Noconspiracy, however, can be defined in different strength. And this is the point where the principles faring worse at the beginning can catch up. Even though Einstein's Reality Criterion provides only separate elements of reality for the correlations, if these elements of reality are strongly non-conspiratorial, then they suffice to derive the Bell inequalities. In short, no-conspiracy together with joint elements of reality and strong no-conspiracy together with separate elements of reality fare equally well in the derivation of the Bell inequalities.

\section{Appendix}

Proof. It is an elementary fact of arithmetic that for any $\alpha, \alpha^{\prime}, \beta, \beta^{\prime} \in[0,1]$ we have

$$
-1 \leqslant \alpha \beta+\alpha \beta^{\prime}+\alpha^{\prime} \beta-\alpha^{\prime} \beta^{\prime}-\alpha-\beta \leqslant 0
$$

Now, let $\alpha, \alpha^{\prime}, \beta, \beta^{\prime}$ be

$$
\begin{aligned}
\alpha & =p\left(A_{i} \mid a_{i} \wedge C_{k}\right) \\
\alpha^{\prime} & =p\left(A_{i^{\prime}} \mid a_{i^{\prime}} \wedge C_{k}\right) \\
\beta & =p\left(B_{j} \mid b_{j} \wedge C_{k}\right) \\
\beta^{\prime} & =p\left(B_{j^{\prime}} \mid b_{j^{\prime}} \wedge C_{k}\right)
\end{aligned}
$$

Substituting (41)-(44) into (40) we get

$$
\begin{array}{r}
-1 \leqslant p\left(A_{i} \mid a_{i} \wedge C_{k}\right) p\left(B_{j} \mid b_{j} \wedge C_{k}\right)+p\left(A_{i} \mid a_{i} \wedge C_{k}\right) p\left(B_{j^{\prime}} \mid b_{j^{\prime}} \wedge C_{k}\right) \\
+p\left(A_{i^{\prime}} \mid a_{i^{\prime}} \wedge C_{k}\right) p\left(B_{j} \mid b_{j} \wedge C_{k}\right)-p\left(A_{i^{\prime}} \mid a_{i^{\prime}} \wedge C_{k}\right) p\left(B_{j^{\prime}} \mid b_{j^{\prime}} \wedge C_{k}\right) \\
-p\left(A_{i} \mid a_{i} \wedge C_{k}\right)-p\left(B_{j} \mid b_{j} \wedge C_{k}\right) \leqslant 0
\end{array}
$$


Using the screener-off condition (28) we obtain

$$
\begin{array}{r}
-1 \leqslant p\left(A_{i} \wedge B_{j} \mid a_{i} \wedge b_{j} \wedge C_{k}\right)+p\left(A_{i} \wedge B_{j^{\prime}} \mid a_{i} \wedge b_{j^{\prime}} \wedge C_{k}\right) \\
+p\left(A_{i^{\prime}} \wedge B_{j} \mid a_{i^{\prime}} \wedge b_{j} \wedge C_{k}\right)-p\left(A_{i^{\prime}} \wedge B_{j^{\prime}} \mid a_{i^{\prime}} \wedge b_{j^{\prime}} \wedge C_{k}\right) \\
-p\left(A_{i} \mid a_{i} \wedge C_{k}\right)-p\left(B_{j} \mid b_{j} \wedge C_{k}\right) \leqslant 0
\end{array}
$$

Multiplying by $p\left(C_{k}\right)$, using no-conspiracy (29) and summing up for $k$ one arrives at (30).

Acknowledgements. I wish to thank Márton Gömöri and Balázs Gyenis for valuable discussions. This work has been supported by the Hungarian Scientific Research Fund, OTKA K-115593.

\section{References}

J. S. Bell, "On the Einstein-Podolsky-Rosen paradox," Physics, 1, 195-200 (1964); reprinted in (Bell 2004, 14-21).

J. S. Bell, "La nouvelle cuisine," in: J. Sarlemijn and P. Kroes (eds.), Between Science and Technology, Elsevier, (1990); reprinted in (Bell 2004, 232-248).

J. S. Bell, Speakable and Unspeakable in Quantum Mechanics, (Cambridge: Cambridge University Press, 2004).

A. Einstein, B. Podolsky and N. Rosen, "Can Quantum Mechanical Description of Physical Reality be considered complete?," Phys. Rew., 47, 777-780 (1935).

A. Fine, The Shaky Game, Einstein, Realism and the Quantum Theory, (Chicago: University of Chicago Press, 1996).

M. Gömöri and G. Hofer-Szabó, "On the meaning of EPR's Criterion of Reality," (in preparation, 2017).

S. Goldstein, T. Norsen, D. V. Tausk, and N. Zanghi, "Bell's theorem," Scholarpedia, 6(10), 8378 (2011).

A. Hájek and J. Bub, "EPR," Found. Phys., 22, 313-331 (1992).

G. Hofer-Szabó, M. Rédei and L. E. Szabó, The Principle of the Common Cause, (Cambridge: Cambridge University Press, 2013).

G. Hofer-Szabó and P. Vecsernyés, "A generalized definition of Bell's local causality," Synthese 193(10), 3195-3207 (2016).

D. Howard, "Einstein on Locality and Separability," Stud. Hist. Phil. Sci., 16, 171201 (1985).

P. J. Lewis, "Bell's theorem, realism, and locality," URL = http://philsci-archive. pitt.edu/11372/ (2015).

T. Maudlin, "What Bell did," J. Phys. A: Math. Theor., 47, 424010 (2014).

J. D. Norton, Einstein for Everyone, URL = http://www.pitt.edu/ jdnorton/ teaching/HPS_0410/index.html

M. Redhead, Incompleteness, Nonlocality, and Realism, (Oxford: Clarendon Press, 1987).

L. E. Szabó, "The Einstein-Podolsky-Rosen Argument and the Bell Inequalities," Internet Encyclopedia of Philosophy, URL= http://www.iep.utm.edu/epr/ (2008). 